\title{
Free energy and stiffness of $\langle 110\rangle$ and $\langle 010\rangle$ steps on a (001) surface of a cubic lattice: Revival of the solid-on-solid model
}

\author{
Harold J. W. Zandvliet, Ronny Van Moere, and Bene Poelsema \\ Solid State Physics Group and MESA + Research Institute, University of Twente, P.O. Box 217, 7500 AE Enschede, The Netherlands
}

(Received 25 April 2003; published 27 August 2003)

\begin{abstract}
We have determined the stiffness and free energy of $\langle 110\rangle$ and $\langle 010\rangle$ oriented steps on a (001) surface of a cubic lattice within the framework of a solid-on-solid model having nearest-neighbor (NN) and next-nearestneighbor (NNN) interactions. The stiffness of $\langle 110\rangle$ oriented steps depends on both the strength of the NN and NNN interactions, whereas the stiffness of the $\langle 010\rangle$ oriented steps only depends on the NNN interaction. For a vanishing NNN interaction the dimensionless inverse stiffness of the $\langle 010\rangle$ oriented steps reduces to the universal value of $1 / \sqrt{2}$ as obtained by the Ising model. As an example we apply this solid-on-solid model to the well-studied $\mathrm{Cu}(001)$ surface.
\end{abstract}

DOI: 10.1103/PhysRevB.68.073404

PACS number(s): 68.35.Md, 05.70.Np, 81.10.Aj

\section{INTRODUCTION}

The free energy of monatomic steps on single-crystal surfaces is a fundamental parameter to describe many surface processes. $^{1-3}$ The thermal roughening temperature of a facet of a crystal is determined by the step free energy. At zero temperature the step free energy is equal to the step formation energy. With increasing temperature, however, the step free energy decreases to a meandering of the step. Although step meandering requires kink creation, the entropy gain is sufficient to result in an overall decrease of the step free energy. One can easily show that an isolated step can always lower its free energy at nonzero temperatures by meandering. Thermodynamically an isolated step is therefore always rough at temperatures above $0 \mathrm{~K}$. A facet will thermally roughen at the temperature at which the step free energy vanishes.

Besides the roughening of a facet the step free energy also determines the two-dimensional (2D) equilibrium shape of an island or vacancy island on a crystal surface. Within the framework of the two-dimensional Ising model Rottman and Wortis ${ }^{4}$ determined the exact equilibrium shape of an island. They found that in the case of isotropic nearest-neighbor interactions the equilibrium shape at $0 \mathrm{~K}$ is a perfect square, whereas the corners of the square become rounded at higher temperatures.

Although we have only briefly discussed a few equilibrium surface processes there are many nonequilibrium processes, such as crystal growth and etching, in which the step free energy plays a leading role. Besides the step free energy there is another quantity, denoted as step stiffness, which is often used to describe the meandering of a step. The step stiffness describes the resistance to meandering of the step and is closely related to the energy required to generate a kink in the step. Within the framework of a simple solid-onsolid model with only nearest-neighbor (NN) interactions, expressions for the step free energy and step stiffness for various types of high-symmetry steps, such as, for instance, $\langle 110\rangle$ and $\langle 010\rangle$ oriented steps on a simple cubic (001) surface, have already been derived. ${ }^{4,5}$ Here we consider a slightly more complicated solid-on-solid model that includes a next-nearest-neighbor (NNN) (diagonal) interaction as well. For semiconductors surfaces, for which step and kink energies can be associated with covalent bonds, this method works very satisfactorily. ${ }^{2,6-9}$ In contrast to semiconductors, metals have wave functions that are quite extended in comparison to the spacing between nearest-neighbor atoms. Therefore it is natural to assume that in the case of metals, NNN and perhaps even next-next-nearest-neighbor interactions might be needed to describe the energetics of the surface properly. If we restrict ourselves to NN and NNN interactions only we show that the stiffness of the $\langle 110\rangle$ oriented step depends on both the NN and NNN interactions, whereas the stiffness of $\langle 010\rangle$ oriented steps only depends on the NNN interaction. Expressions for the step free energies are also derived.

As an illustrative example we show that within the framework of the solid-on-solid model that includes NN and NNN interactions, the energetics of $\mathrm{Cu}(001)$ can be described adequately. The difference between the kink creation energy (130 meV/a) (Refs. 10-12) and the [110] step formation energy (220 meV/a) (Refs. 13 and 14) can simply be understood in terms of NN and NNN interaction energies. Moreover, the temperature dependence of the 2D island equilibrium shape and the high stiffness of a $\langle 010\rangle$ oriented step on $\mathrm{Cu}(001)$ are also described reasonably well.

\section{SOLID-ON-SOLID MODEL WITH NN AND NNN INTERACTIONS}

The meandering of a step can be characterized by the spatial step correlation function $G(x)$, which is defined as ${ }^{15}$

$$
G(x)=\left\langle\left[y\left(x+x_{0}\right)-y\left(x_{0}\right)\right]^{2}\right\rangle=\left\langle n^{2}\right\rangle \frac{|x|}{a_{\|}},
$$

where $x$ and $y$ are coordinates parallel and perpendicular to the mean step edge direction, while $a_{\|}$and $a_{\perp}$ are the atomic units along and perpendicular to the step edge. Finally, $\left\langle n^{2}\right\rangle$ refers to the mean-square kink length and is defined as ${ }^{16}$

$$
\left\langle n^{2}\right\rangle=\sum_{i} n_{i}^{2} p_{i}
$$



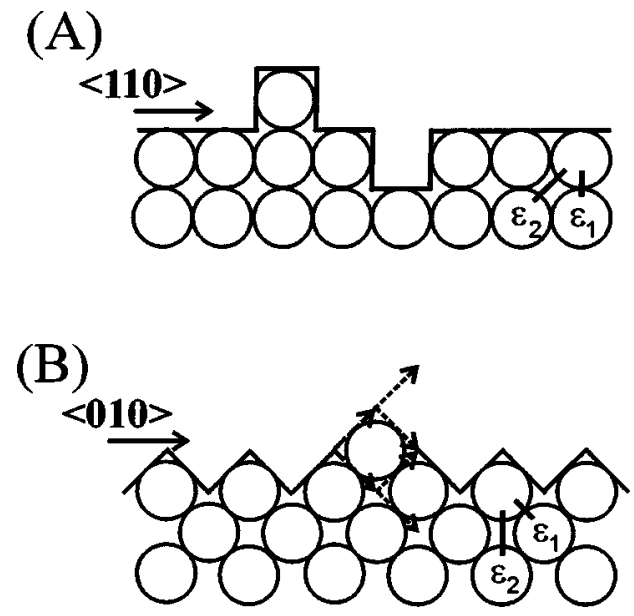

FIG. 1. Schematic diagrams of (a) $\langle 110\rangle$ and (b) $\langle 010\rangle$ oriented steps on a (001) surface of a cubic crystal. $\varepsilon_{1}$ and $\varepsilon_{2}$ refer to the NN and NNN interaction energies between the surface atoms. The NN and NNN distances between atoms are $a$ and $\sqrt{2} a$, respectively.

where $p_{i}$ refers to the probability of finding a kink with a length of $n_{i}$ units. The summation runs over all possible kink configurations. The mean-square kink length is the expectation value of the square kink length at each step edge position and can be considered as the diffusivity of the step edge. At zero temperature (or infinity large kink creation energy) the step edge diffusivity or mean-square kink length is reduced to zero. The spatial step correlation function can also be written in terms of the step edge stiffness $\gamma:{ }^{14}$

$$
G(x)=\left\langle\left[y\left(x+x_{0}\right)-y(x)\right]^{2}\right\rangle=\frac{k_{b} T}{\gamma a_{\|}} a_{\|}|x|,
$$

where the dimensionless inverse step edge stiffness is defined as

$$
\frac{k_{b} T}{\gamma a_{\|}}
$$

where $T$ is the temperature and $k_{b}$ Boltzmann's constant. Thus the following relation between the step edge stiffness and mean-square kink length exists,

$$
\frac{k_{b} T}{\gamma a_{\|}}=\frac{\left\langle n^{2}\right\rangle}{a_{\|}^{2}} .
$$

First, we consider a step running along a $\langle 110\rangle$ direction (see Fig. 1). Within the framework of a solid-on-solid model with NN $\left(\varepsilon_{1}\right)$ and NNN $\left(\varepsilon_{2}\right)$ interactions the step edge formation energy per unit length $a_{\|}(=a)$ is given by ${ }^{2}$

$$
E_{\langle 110\rangle}=\frac{\varepsilon_{1}}{2}+\varepsilon_{2}
$$

The kink formation energy of a kink with a length of $n$ ( $n$ $\geqslant 1$ ) units is ${ }^{2}$

$$
E_{k}(n)=n \frac{\varepsilon_{1}}{2}+(n-1) \varepsilon_{2} .
$$

Instead of a set of $\mathrm{NN}$ and NNN interaction energies it is equally valid to consider a NN interaction energy $(\chi)$ and an effective corner energy $(\kappa)$. The following relations between the two sets of interaction energies can be derived: ${ }^{6,7}$

$$
\begin{gathered}
\chi=\varepsilon_{1}+2 \varepsilon_{2}, \\
\kappa=-\varepsilon_{2} .
\end{gathered}
$$

This set of interaction energies also result in a step edge and single kink formation energies of 220 and $130 \mathrm{meV} / a$, respectively.

The step free energy per unit length $a$ is given by

$$
F=-k_{b} T \ln Z=-k_{b} T \ln \left(\sum_{i} e^{-E_{i} / k_{b} T}\right),
$$

where the summation runs over all possible step configurations and $E_{i}$ refers to the formation energy of the $i$ th configuration. The partition function of a $\langle 110\rangle$ oriented step, $Z_{\langle 110\rangle}$, is given $b^{2}$

$$
Z_{\langle 110\rangle}=e^{-E_{\langle 110\rangle} / k_{b} T}\left[1+2 \sum_{n=1}^{\infty} e^{-E_{k}(n) / k_{b} T}\right] .
$$

The step free energy per unit length $a$ of a $\langle 110\rangle$ oriented step is then

$$
F_{\langle 110\rangle}=\frac{\varepsilon_{1}}{2}+\varepsilon_{2}-k_{b} T \ln \left[1+\frac{2 e^{-\varepsilon_{1} / 2 k_{b} T}}{1-e^{-\left(\varepsilon_{1} / 2+\varepsilon_{2}\right) / k_{b} T}}\right] .
$$

The mean-square kink length can now easily be calculated to be

$$
\left\langle n^{2}\right\rangle=\frac{2 a_{\perp}^{2} \sum_{n=1}^{\infty} n^{2} e^{-E_{k}(n) / k_{b} T}}{1+2 \sum_{n=1}^{\infty} e^{-E_{k}(n) / k_{b} T}} .
$$

Substituting the kink formation energy and some mathematics finally results in

$$
\left\langle n^{2}\right\rangle_{\langle 110\rangle}=\frac{2 a_{\perp}^{2} e^{\varepsilon_{2} / k_{b} T} e^{-\left(\varepsilon_{1} / 2+\varepsilon_{2}\right) / k_{b} T}\left[1+e^{-\left(\varepsilon_{1} / 2+\varepsilon_{2}\right) / k_{b} T}\right]}{\left[1-e^{-\left(\varepsilon_{1} / 2+\varepsilon_{2}\right) / k_{b} T}+2 e^{-\left(\varepsilon_{1} / 2+\varepsilon_{2}\right) / k_{b} T} e^{\varepsilon_{2} / k_{b} T}\right]\left[1-e^{-\left(\varepsilon_{1} / 2+\varepsilon_{2}\right) / k_{b} T}\right]^{2}}
$$

and thus

$$
\frac{k_{b} T}{\gamma_{\langle 110\rangle} a}=\frac{2 e^{-\varepsilon_{1} / 2 k_{b} T}\left[1+e^{-\left(\varepsilon_{1} / 2+\varepsilon_{2}\right) / k_{b} T}\right]}{\left[1-e^{-\left(\varepsilon_{1} / 2+\varepsilon_{2}\right) / k_{b} T}+2 e^{-\varepsilon_{1} / 2 k_{b} T}\right]\left[1-e^{-\left(\varepsilon_{1} / 2+\varepsilon_{2}\right) / k_{b} T}\right]^{2}} .
$$


Hence the mean-square kink length and the step edge stiffness of a $\langle 110\rangle$ oriented step depend both on the strength of the NN and NNN interactions. As we show below this is quite different for a step oriented along a $\langle 010\rangle$ direction.

Second, we consider a $\langle 010\rangle$ oriented step (see Fig. 1). The route we follow to extract the mean-square kink length, step free energy, and step edge stiffness is analogous to the $\langle 110\rangle$ step. The partition function of a $\langle 010\rangle$ oriented step is given by

$$
Z_{\langle 010\rangle}=e^{-\left(\varepsilon_{1}+\varepsilon_{2}\right) / k_{b} T}+2 e^{-\left(\varepsilon_{1}+3 \varepsilon_{2} / 2\right) / k_{b} T}+e^{-\left(\varepsilon_{1}+2 \varepsilon_{2}\right) / k_{b} T} .
$$

Here we have considered a line segment $a_{\|}=\sqrt{2} a$ along the mean $\langle 010\rangle$ direction. The step edge free energy per unit length $a$ of a $\langle 010\rangle$ oriented step is given by ${ }^{17}$

$$
F_{\langle 010\rangle}=\sqrt{2}\left[\left(\frac{\varepsilon_{1}+\varepsilon_{2}}{2}\right)-k_{b} T \ln \left(1+e^{-\varepsilon_{2} / 2 k_{b} T}\right)\right] .
$$

The mean-square kink length is

$$
\begin{aligned}
\left\langle n^{2}\right\rangle_{\langle 010\rangle} & =\frac{a_{\perp}^{2}\left[e^{-\left(\varepsilon_{1}+2 \varepsilon_{2}\right) / k_{b} T}+e^{-\left(\varepsilon_{1}+3 \varepsilon_{2} / 2\right) / k_{b} T}\right]}{Z_{\langle 010\rangle}} \\
& =\frac{a_{\perp}^{2} e^{-\varepsilon_{2} / 2 k_{b} T}}{1+e^{-\varepsilon_{2} / 2 k_{b} T}} .
\end{aligned}
$$

Because $a_{\perp}=a_{\|}=\sqrt{2} a$ we finally find

$$
\frac{k_{b} T}{\gamma_{\langle 010\rangle} a}=\frac{\sqrt{2}}{1+e^{\varepsilon_{2} / 2 k_{b} T}} .
$$

For a vanishing NNN interaction the dimensionless inverse step stiffness reduces to the universal value $(1 / \sqrt{2})$ of the Ising model. ${ }^{4,9}$ With an increasing attractive NNN interaction the dimensionless inverse step stiffness decreases and thus the step stiffness increases.

\section{APPLICATION TO THE Cu(001) SURFACE}

In order to test the applicability of our model we make contact with the well-studied $\mathrm{Cu}(001)$ surface. The creation energy for a single kink in a $\langle 110\rangle$ oriented step has been determined experimentally by analyzing the spatial step fluctuations (128 $\pm 3 \mathrm{meV} / \mathrm{a})$ (Refs. 10 and 11) and the mini- mum curvature of an island $(131 \pm 9 \mathrm{meV} / a) .{ }^{12}$ The formation energy of a $\langle 110\rangle$ oriented step is extracted from the island equilibrium shape $(220 \pm 20 \mathrm{meV} / a)$ (Ref. 13) and the size dependence of island shape fluctuations (220 $\pm 11 \mathrm{meV} / a) .{ }^{14}$ By using Eqs. (6) and (7) we can extract a $\mathrm{NN}$ interaction energy of $260 \mathrm{meV} / a$ and a NNN (diagonal) interaction energy of $90 \mathrm{meV} /$ corner. Using these values we find a dimensionless inverse stiffness of a $\langle 010\rangle$ oriented step of 0.2 at room temperature, which agrees perfectly with the experimental available data. ${ }^{14}$ However, in order to explain the temperature dependence of the $2 \mathrm{D}$ equilibrium island shape properly, a lower NNN interaction energy has been found of $63 \mathrm{meV} /$ corner. $^{17}$ A NNN interaction energy of 63 $\mathrm{meV} /$ corner results in a dimensionless inverse step stiffness of 0.3 , which is 0.1 higher than the experimentally determined value of 0.2. As suggested by Dieluwiet et al. ${ }^{14}$ it might very well be that more sophisticated many-body interactions such as, for instance, kink-kink interactions contribute to the reduction of the dimensionless inverse step stiffness as well. Because an (attractive) NNN interaction $\left(\varepsilon_{2}\right)$ can be interpreted as a (repulsive) corner energy $(\kappa),{ }^{7} \mathrm{Eq}$. (18) can be used immediately. By inserting $\kappa=-\varepsilon_{2}=$ $-90 \mathrm{meV} /$ corner we arrive again at a dimensionless inverse step edge stiffness of 0.2 , in perfect agreement with the experiments. ${ }^{14}$ Thus, in marked contrast to Ref. 14 we arrive at the conclusion that a NNN interaction (or equivalently, an effective corner energy) is the main cause for the strong reduction of the dimensionless inverse stiffness of a $\langle 010\rangle$ oriented step on $\mathrm{Cu}(001)$.

We have not taken into account additional contributions to the step free energy arising from vibrational entropy terms. ${ }^{18-20}$ Due to the reduced coordination of steps atoms in a $\langle 010\rangle$ oriented versus a $\langle 110\rangle$ oriented step this vibrational entropy term is expected to be larger for $\langle 010\rangle$ than for $\langle 110\rangle$ oriented steps.

\section{CONCLUSIONS}

Within the framework of a solid-on-solid model with NN and NNN interactions we have derived expressions for the step edge stiffness, step free energy, and mean-square kink length of $\langle 110\rangle$ and $\langle 010\rangle$ oriented steps of a (001) surface of a cubic lattice. The energetics of $\mathrm{Cu}(001)$ is described in a very satisfactory way by this solid-on-solid model.
${ }^{1}$ E. D. Williams and N. C. Bartelt, Science 251, 393 (1991).

${ }^{2}$ H. J. W. Zandvliet, Rev. Mod. Phys. 72, 593 (2000).

${ }^{3}$ H.-C. Jeong and E. D. Williams, Surf. Sci. Rep. 34, 171 (1999).

${ }^{4}$ C. Rottman and M. Wortis, Phys. Rev. 24, 6274 (1981).

${ }^{5}$ G. Schulze Icking-Konert, M. Giesen, and H. Ibach, Phys. Rev. Lett. 83, 3880 (1999).

${ }^{6}$ B. S. Swartzentruber, Y.-W. Mo, R. Kariotis, M. G. Lagally, and M. B. Webb, Phys. Rev. Lett. 65, 1913 (1990).

${ }^{7}$ H. J. W. Zandvliet, H. B. Elswijk, E. J. van Loenen, and D. Dijkkamp, Phys. Rev. B 45, 5965 (1992).
${ }^{8}$ E. J. Heller, Z. Y. Zhang, and M. G. Lagally, Phys. Rev. Lett. 71, 743 (1993).

${ }^{9}$ H. J. W. Zandvliet, Phys. Rev. B 61, 9972 (2000).

${ }^{10}$ M. Giesen-Seibert and H. Ibach, Surf. Sci. 326, 205 (1994).

${ }^{11}$ M. Giesen-Seibert, F. Schmitz, R. Jentjens, and H. Ibach, Surf. Sci. 329, 47 (1995).

${ }^{12}$ M. Giesen, C. Steimer, and H. Ibach, Surf. Sci. 471, 80 (2001).

${ }^{13}$ C. Steimer, M. Giesen, L. Verheij, and H. Ibach, Phys. Rev. B 64, 085416 (2001)

${ }^{14}$ S. Dieluwiet, H. Ibach, M. Giesen, and T. L. Einstein, Phys. Rev. B 67, 121410(R) (2003). 
${ }^{15}$ H. J. W. Zandvliet, H. Wormeester, D. J. Wentink, A. Van Silfhout, and H. B. Elswijk, Phys. Rev. Lett. 70, 2122 (1993).

${ }^{16}$ H. J. W. Zandvliet and H. B. Elswijk, Phys. Rev. B 48, 14269 (1993).

${ }^{17}$ R. Van Moere, H. J. W. Zandvliet, and B. Poelsema, Phys. Rev. B
67, 193407 (2003).

${ }^{18}$ J. W. M. Frenken and P. Stoltze, Phys. Rev. Lett. 82, 3500 (1999).

${ }^{19}$ H. P. Bonzel and E. Emundts, Phys. Rev. Lett. 84, 5804 (2000).

${ }^{20}$ H. J. W. Zandvliet, O. Gurlu, and B. Poelsema, Phys. Rev. B 64, 073402 (2001). 